\title{
ASSESSING THE LONG-TERM IMPACT OF A PROFESSIONAL DEVELOPMENT PROGRAM
}

\author{
Marcia M. Tennill, Margaret W. Cohen \\ University of Missouri-St. Louis
}

This study was designed to explore the long-term impact of a year-long faculty development program on participants. Three guiding questions focused the study: In what ways did the program influence the professional lives of participants five years after completion? How did the participants integrate those experiences into their professional lives? and What recommendations for best practices in the field of faculty development can be drawn? Donald Kirkpatrick's four-level evaluation model was the template for this qualitative research. Results indicated that participants retained program learning over time.

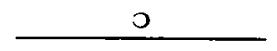

This program evaluation explored the long-term impact of faculty development in higher education and is situated in the context of a specific program: the University of Missouri's New Faculty Teaching Scholars Program (NFTS). Although this research was based on the experiences of faculty who participated in that specific program, it also broadly addressed the concept of faculty development and its general 
relevance to higher education. Faculty development programs in higher education broadly refer to activities that focus on enhancing the professional success of faculty members as teachers, scholars, professionals, and individuals. While each of these areas is valuable and worthy of study, exploring all of them presented a task that exceeded the goals of the research. Instead, data collection addressed four of the five NFTS programmatic goals that were directed at supporting the faculty member's teaching role.

\section{New Faculty Teaching Scholars Program}

The 2001 strategic plan of the University of Missouri (UM) called for the development of campus environments focused intentionally on student learning. One response was the systemwide NFTS. Initiated in the 20012002 academic year, NFTS continued annually until it was suspended for budgetary reasons during the 2008-2009 academic year. The program was administered through the UM system office of academic affairs and coordinated by a director on each of the four campuses (Columbia, St. Louis, Kansas City, and Rolla). New and early-career faculty members in their second, third, or fourth years of appointment were eligible to participate. At the time of application, both the faculty member and the department chair acknowledged in writing the time commitment necessary to participate fully in the program. Approximately fifty faculty members participated annually.

The program acclimated new faculty members to their roles and responsibilities on their own campuses and across the university system. As noted in the program brochure (New Faculty Teaching Scholars, 2004), the NFTS had these goals:

1. To support individual campus activities that help new faculty members become effective teachers and scholars.

2. To promote engaged instructional strategies that support student-centered learning environments.

3. To assist in the development of campus and systemwide networks that are essential for success in today's academic world.

4. To support increased faculty research, teaching productivity and faculty retention.

5. To support efforts to develop a "culture of teaching" on each campus and throughout the University of Missouri. 
The NFTS program spanned the nine-month academic year. Three two- to three-day systemwide retreats were scheduled-one each fall, winter, and spring. Two of the retreats convened in locations removed from each of the campuses. Expenses associated with travel, accommodations, meals, and materials were funded by the UM System. The fall retreat focused on course design and offered participants opportunities to learn about, develop, and apply active and learner-centered teaching strategies to their courses. The winter retreat was held concurrently with a teaching renewal conference on the Columbia campus, where NFTS participants participated in conference workshops and instructional presentations facilitated by experts in the fields of teaching and learning. The spring retreat focused participants on creating an academic portfolio describing and documenting their academic accomplishments and professional development. The portfolio serves as a foundation for promotion and tenure dossiers. In addition, each director sponsored monthly presentations and discussions for his or her campus's participants. Although these events were scheduled to reflect the NFTS goals, directors had the flexibility to tailor events to meet the needs of their campus group. For example, events on the Rolla campus, now Missouri University of Science and Technology, might focus programs on its research mission, devoting attention to National Science Foundation funding.

Thus, NFTS convened the faculty participants multiple times during the year as a single cohort and as campus cohort groups. University administrators and the program directors hoped to nurture a culture of teaching across the university system. They sought to foster environments where early-career faculty engaged with students and one another in learner-centered environments. The program was designed to expand faculty members' collegial networks by creating relationships that might lead to joint research or teaching collaborations. Ultimately administrators wanted NFTS to engage, encourage, and entice early-career faculty to stay at the university until they attained tenure and beyond.

Based on the strategic plan set forth by the University of Missouri System (Pacheco, 2001) and the goals of the NFTS program (New Faculty Teaching Scholars, 2004), the yearly evaluations of the NFTS program generally reflected outcomes found in the literature. At the end of each program year, participants completed a comprehensive evaluation of the activities, the content, and the perceived effect of the program on their professional development. During the seven years of the program, 395 faculty members attended and completed the program. Of those faculty participants, 74 percent responded to the end-of-program evaluative surveys. Those participants agreed that NFTS had a positive impact on 
their teaching (average agreement, 89 percent), their collegial relationships (average agreement, 89 percent), and their professional development as related to promotion and tenure (average agreement, 57 percent for the three years that issue was surveyed). These data are compiled from the annual evaluation reports from each program year (University of Missouri System, 2001-2008).

Nevertheless, a larger question remained: "How does this program impact the professional lives of participants on a long-term basis?" One means of determining long-term program impact is to follow up with participants after a period of time has passed.

\section{Guiding Questions}

Qualitative research methods explore the details of selected issues in depth. The purpose of this research study, then, was not to measure, compare, or quantify outcomes of the program but to explore with participants how and in what ways the program has affected them on a longterm basis. The following guiding questions served as the template for this qualitative research study:

1. In what ways has NFTS influenced the professional lives of participants five years after completing the program?

2. How have participants integrated NFTS experiences into their professional lives?

3. How do the results of this study offer recommendations for best practices in the field of faculty development?

\section{Review of the Literature}

Faculty development is now regarded as integral to higher education. Broadly, the term is used to describe activities that focus on the multiple roles of faculty in the current educational environment. These roles encompass teaching, research, scholarship, and service within the educational system and in the community (Amundsen et al., 2005). The literature subsumes many documented evaluations of faculty development programs, and most reported outcomes are positive. Faculty who participated generally found them helpful in several areas. They appreciated the opportunities to learn and implement classroom strategies that contribute to improved teaching and relevant learning for their students. Participants reported that their teaching skills improved (Camblin \& Steger, 2000; Davidson-Shivers, Salazar, \& Hamilton, 2005; Pittas, 2000), and they were more confident and satisfied with their teaching 
(Knight, Carrese, \& Wright, 2007). They also valued the collegial relationships that often developed through these programs. Participants established social and professional relationships with peers and mentors (Morzinski \& Fisher, 2002; Pittas, 2000), created cooperative partnerships across disciplines (Camblin \& Steger, 2000), and had stronger personal communication with colleagues (Knight et al., 2007).

\section{Importance of Follow-Up Evaluation}

The majority of faculty development program evaluations stop with a one-time assessment on the completion of a program, whether it was a one-day workshop or a year-long series. However, there is a persistent call in the literature for long-term evaluations of faculty development programs (Knight et al., 2007; Morzinski \& Simpson, 2003; Steinert, 2000). For the purposes of this study, the terms follow-up evaluation and long-term evaluation are used interchangeably to indicate an evaluation that takes place after a given period of time has passed after program completion.

While it is essential and often valuable to get a snapshot assessment of the impact of a program at its conclusion, it is equally vital to explore the long-term effects of a program on participants to see if and how they are transferring recently acquired skills and knowledge to their current professional situations. For example, initial effects of training may fade over time if participants are no longer in an ideal and supportive environment. In other instances, there may be a delay in how participants implement newly acquired knowledge. Some participants may not report an immediate impact of a program but may realize important positive outcomes at a later date. Consequently, a follow-up evaluation of a program not only explores the continuing effects of a program but also has the potential to identify outcomes experienced only after the program has ended. This program evaluation was a formal, summative exploration of the impact of NFTS on participants five years after completing the program. We based our design of this evaluation on Kirkpatrick's (1975) four-level model of training evaluation.

\section{Methodology}

Based on the study's purpose and the questions guiding our inquiry, qualitative methods were ideal for this study. While quantitative methods concentrate on testing specific hypotheses (Worthen, Sanders, \& Fitzpatrick, 1997) and generally seek "explanations and predictions that will generalize to other persons and places" (Thomas, 2003, p. 2), qualitative 
methods, including qualitative evaluations, "permit the evaluator to study selected issues in depth and detail" (Patton, 1990, p. 13). Patton suggests that studying a small number of cases enables qualitative researchers to generate detailed information that can lead to a better understanding of the cases or of the topic being studied. Based on the guiding questions, this study's purpose was not to predict or generalize the impact of the NFTS program to other populations, but rather to explore in detail what types of impacts the program had on participants and how those program experiences affected participants' professional lives. Therefore, qualitative methodology was the appropriate choice for this study.

Document review, interviews, and observations are inherent sources of data for qualitative research and evaluation (Patton, 1990). These three methods provided information to meet all four levels of the evaluation model, and each was incorporated into the design. We used purposeful random sampling to select twelve participants from two program years and interviewed them to learn how the NFTS program had affected them over the long term. We also reviewed CVs and one course syllabus for each participant and identified professional activities that reflected and supported their interview responses. We observed in the classrooms of two participants from the study. These data points served as a triangulation of the data sources. Interviews with selected participants, review of their CVs and course syllabi, and classroom observations provided evidence of the impact the NFTS program had on their professional lives and how that initial impact continued to affect their professional behaviors.

\section{Kirkpatrick's Model}

Kirkpatrick's (1975) model of training evaluation offers a standard for evaluating industry and business training programs. It is based on four levels of measurement: reaction, learning, behavior, and results. Kirkpatrick notes that measuring any one of these program stages is informative, but he argues that an evaluation of all four levels provides a more complete picture of a program's effectiveness.

Kirkpatrick's (1998) model has been used successfully to evaluate training programs in business and industry and is regarded as applicable to academic evaluations (Boyle \& Crosby, 1997; Naugle, Naugle, \& Naugle, 2000). Morzinski and Simpson (2003) applied the four-level model to evaluate longitudinal outcomes of a faculty development program for family medical practitioners. Their application of all four levels illustrates its applicability to our study. 
We addressed the guiding questions of this research using Kirkpatrick's model in the following manner:

1. Reaction: What did the selected participants think about the retreats and campus events? We reviewed and summarized the annual evaluation reports from the two years selected to gather information reflective of participants' perceptions about the program. During the interviews, we gathered retrospective data when we asked participants to offer their thoughts about their year of participation in the NFTS program.

2. Learning: What did the selected participants learn? Did the selected participants learn more about teaching? Did their attitudes about teaching change? Did they learn additional skills? Participant responses to these questions were gathered as we reviewed and summarized annual evaluation reports from the two years selected for this study. During interviews, we asked study participants to reflect on and discuss what they had learned from the program.

3. Behavior: How has the professional behavior of the selected participants changed? How is it different? Did it change in the classroom? Did it change in relation to peers? Did it change their professional activities? This level of the evaluation model was addressed during in-depth interviews with study participants. We also reviewed their CVs and a syllabus and observed in several classrooms.

4. Results: How has the NFTS program affected the selected participants, their departments, their campus, or the university? This level of the evaluation model was probed during individual, in-depth interviews conducted with each participant.

\section{Participants and Sample Size}

We relied on a purposeful random sample of study participants. Randomly selecting participants from even a small population indicates that the researcher is reporting data in advance of knowing outcomes (Patton, 1990). Patton argues that it is critical to understand that this is a purposeful random sample, not a representative random sample. This type of small random sample offers credibility to the data, not representativeness or generalization to other populations.

We invited faculty members who completed the NFTS program during the 2004 and 2005 academic years to participate. Approximately 50 faculty members participated in the NFTS program each academic year, 
and targeting these two program years yielded 106 faculty members as potential participants. Since face-to-face interviews and classroom observations were part of data collection, only the 89 faculty participants who were still working on a UM campus were included.

Three participants from each of the four UM campus NFTS groups were randomly selected as potential participants, resulting in a sample size of twelve. While there is minimal guidance in the literature regarding the number of interviews that are ideal for a qualitative research project, guidance came from an extensive review of the academic and medical literature conducted by Guest, Bunce, and Johnson (2006). Their study on data saturation in qualitative interviewing concluded that twelve interviews generally suffice when a researcher's intention is to explore common perceptions and experiences of a relatively homogeneous group of individuals. This reinforced our reasoning to include twelve participants in this study.

Males and females were evenly represented in the sample. Their teaching experience ranged from teaching assistant experience to more than nine years of full-time teaching. They represented twelve different departments and professional programs. Six had attained tenure, and three were tenure-track assistant professors. Two were in nontenure-track positions, and one had already committed to moving to a position outside the UM system.

\section{Analysis}

Content analyses were used to analyze the results of this study. We relied on inductive analysis techniques, which allowed patterns, themes, and categories to emerge from the data (Patton, 1990). We used document review to analyze existing evaluations from the two NFTS program years selected for study and analyze participant $\mathrm{CVs}$ and syllabi. The interviews were analyzed using cross-case analysis. The interview guide served as an analytical framework for the data. We also used cross-case analysis as a strategy to analyze the classroom observations.

\section{Results}

On all four levels of Kirkpatrick's evaluation model, NFTS produced positive results for most participants.

REACTION The general reaction to the NFTS program was positive. Participant responses on the end-of-year surveys and study participants' 
interview comments mostly reflected satisfaction and an appreciation for the program. The majority of both participant groups welcomed the opportunities provided by NFTS to improve their teaching and expand their peer networks. One person commented:

So it was nice to have space and time dedicated to thinking about teaching. I felt that the program, especially the fall retreat, focusing on active engagement of students and student centered learning, I felt like that was really helpful. Actually, I still have a book they gave us and I pull it out once in a while: active learning. I think that was really great.

Others appreciated the guidance offered for their career advancement and the efforts of the university to welcome them as new faculty. Positive reactions from the study participants were similar to those from surveyed participants four or five years ago. This indicated that positive feelings about the program were basically held constant over time for many of the participants. As Kirkpatrick (1998) stated, those who liked and enjoyed a program have the potential to reap the greatest benefits.

Although there were no surveyed participants who expressed disappointment in the program, three of the study participants had suggestions that reflected a less positive reaction to the program. All three comments reflected a lack of connection with the program on some level. One participant mentioned that he was the only one in his field, another commented that the content presented didn't apply to his educational strategies, and the last stated that he had more teaching experience than the other participants.

LEARNING Most participants from both groups, those who responded to the end-of-year surveys and those who participated in this study, responded in a similar manner when asked what they had learned from the NFTS program. They noted that their attitudes toward teaching shifted to be more active and engaging and identified specific teaching strategies that they incorporated into their classrooms. Some participants learned to modify an existing course strategy, and others learned to implement new ones. One person mentioned:

Specifically, I teach large lecture classes, so I learned how to make them student centered, how to make them active, the think-pairshare thing. I think that was the first year I started using clickers in the classroom, and there was a presentation that gave me some kind of real tangible ways of using the clickers and ways that I hadn't thought about before: using them for opinion-like thought questions instead of 
just attendance or quiz-like questions. It pushed me to use them in ways that I hadn't done before. Now I use them in a completely different way than I used to. Now I use them to gather evidence from the students to demonstrate a theory that we're discussing in class.

According to Kirkpatrick's (1998) model, such positive responses are evidence that these participants did in fact have a learning experience during the program.

Not all comments about what was learned during NFTS were positive. Several survey participants indicated that their NFTS experience resulted in minimal learning. Again, the comments reflected a lack of connection with the program at some level. One participant said the program content dealt with class sizes that did not match his, a few thought the content of the program was too general to apply to their own courses, and another said the program was better suited to inexperienced teachers. One study participant reiterated again that his teaching strategies were very different from those presented at NFTS and that he would continue teaching in that way. It is worth noting that most of the negative comments regarding the program came from the same few participants.

BEHAVIOR Participants in this study mostly agreed that NFTS had changed their professional behaviors in some way. Expanding on the discussions of what they learned in the program, most agreed that their professional behaviors in the classroom have changed to include instructional strategies focused on active and engaged learning. They also mentioned that they benefited from conversations and discussions about teaching strategies with their NFTS peers. Some of those participants said that the strategies they learned and implemented from NFTS had a positive impact on student learning in their classes.

Three of the twelve study participants acknowledged that NFTS had not made any specific difference in their classroom and teaching behaviors. Of those three, the same participant reiterated that his teaching strategies differed from those presented at NFTS, one commented that he would continue teaching based on his previous experience, and the other noted that NFTS had more of an impact on relationships with his peers. The inference of no connection with program content is evident again.

All study participants acknowledged that their social or professional peer networks expanded during the NFTS program year. One participant explained:

I can call my NFTS friend who's in another college and say: "You know, I've got this student here, and this is the situation, this is what 
happened, and this is the argument they're making and this is what I'm thinking. What do you think? Is this appropriate or not?" ... That's the greatest thing about that network, having that support and people you can trust to go to and be vulnerable. That's the big thing, too, is there's ego involved. Having a network with people you can go to and trust and be comfortable with, possibly exposing yourself as an idiot... Most of my networking is peer networking, the NFTS contacts.

Some enjoyed new relationships with colleagues on their home campuses, and others forged collaborations with NFTS colleagues across the UM system. However, several participants lamented that those peer relationships were not sustained after the end of the program year. Ten of the twelve participants agreed that networking was a vital part of their professional lives. They revealed that networking occurred in a variety of ways, but most of them acknowledged that the most productive opportunities to network took place at planned events. Supporting evidence from $\mathrm{CV}$ and syllabi reviews and from classroom observations corroborated the behavior changes discussed by participants. Based on Kirkpatrick's (1998) model, these behavior changes are indications of a positive program effect.

\section{Benefits of the Experience}

The study participants thoughtfully discussed the benefits of their NFTS experiences. On a personal level, some agreed that their instructional knowledge and classroom confidence increased because of NFTS. Others acknowledged that the social and professional relationships developed during NFTS were both enjoyable and beneficial, and a few participants appreciated the guidance offered for their own career advancement. Interestingly, two participants who fully participated and embraced the NFTS program mentioned that their focus on improved teaching would probably delay their promotions, explaining that some aspects of the university culture continue to undervalue teaching.

Most participants recognized that their personal benefits from the program extended into their departments as well. They said that improved and engaged teaching positively affected their departments by attracting and retaining more students. One person commented:

Students that are in dynamic classrooms and have mentoring relationships with faculty are by definition more engaged. More engaged students are going to participate more on campus and they're going to 
have greater allegiances to the university. It's funny, if you ask the students who's the chancellor or who's the provost, they usually don't know, but they can tell you who teaches Psych 101. This is my perspective as a faculty member, but I think faculty are the face of the university for the students, and in many instances, the universe for the students. So if you make faculty more effective in teaching and make them more open to adult learning, then I think the students have a better experience, they're more engaged in the university, and that benefits everyone.

Participants also mentioned that expanded networking broadened and brought a more global perspective to their departments. Some participants noted that these same types of benefits carried over to their home campuses, adding that benefits from NFTS could also have a positive effect on faculty retention. Although most of the participants had difficulty envisioning a connection between NFTS and benefits to the university system, a few added that the positive program outcomes could also contribute to alumni support. These results reflected Kirkpatrick's (1998) model by identifying outcomes that are beneficial on multiple levels.

While it is invaluable to assess a program at its conclusion, it is increasingly important to explore the long-term impact of a program on participants. This allows the evaluator to see if and how program participants are applying recently acquired skills and knowledge to their current professional situation. This study met the call for long-term evaluations of faculty development programs (Knight et al., 2007; Morzinski \& Simpson, 2003; Steinert, 2000), and the results provided evidence that the NFTS program continued to have positive impacts on its participants over time.

\section{Discussion and Recommendations}

This qualitative study offers participants' personal narratives about their program participation. Exploring experiences over time offers insight into how such programs continue to have an impact on the professional lives of faculty. While participants identified behavioral changes in their professional lives following program participation, it is possible that such changes relate to multiple sources. We learn from theories of motivation how difficult it is to attribute change to a single event or experience.

Our results suggest that the success of this faculty development program, and perhaps other similar programs, might share several common themes. Successful programs offer content that is current, relevant, and 
accessible to all participants. The program brought together heterogeneous groups of early-career faculty, offering opportunities to experience and share active and engaged teaching and learning strategies. Program participants came from four campuses and differed in discipline, teaching experience, and preparation. The diversity of participants' experiences enhanced social and professional conversations during the program, but that diversity may also have isolated or hindered several participants from fully experiencing the program. It is ideal to advise program planners to help individual participants access and integrate program content into their own professional lives. However, it is far more practical to remind program planners of their responsibility to be transparent and explicit about the program's objectives and content so that applicants can make informed decisions about participation. For example, why would an experienced teacher opt to participate fully in the program?

We propose that successful programs present opportunities for participants to build relationships on both social and professional levels. This program provided multiple opportunities for discussion and conversations among participants. The structured retreats and topical campus meetings offered opportunities for participants to share and engage in professional and academic conversations. These gatherings also incorporated a social component, which allowed the faculty to broaden their personal networks within the UM system. Participants often mentioned networking as an important benefit of the program.

We also suggest that successful programs provide structured follow-up activities for program participants. Many participants commented on the value of networking and expressed an interest in continued connections with their peers after the conclusion of the program. Program planners could incorporate follow-up events to provide continuing opportunities for program alumni to maintain social and professional relationships with their peers.

The results of this study also pose considerations for future research related to long-term evaluations of faculty development programs. Future studies could use multiple perspectives by expanding data sources to include discussions with students, peers, supervisors, and appropriate campus administrators. Future studies could explore whether collecting data using different methods, such as written or online surveys or phone calls, would yield similar responses. Researchers could explore whether five years after a program is long enough to measure the impact of development programs.

Multiple factors emerged that might have affected the participants' successes in the program. Participants listed a wide range of previous 
teaching experience, training in education, working in a variety of disciplines, and instructional obligations encompassing teaching small graduate classes to large lecture ones. One could investigate how these differences contribute to positive experiences in programs like this one. In addition, researchers could ask whether veterans of such programs are more likely to participate in future faculty development or professional development programs. One could also assess the cultures of campuses that support programs like NFTS to see if those cultures are shifting to a system that appreciates and rewards teaching activities. One could also explore whether programs like this result over time in more effective teachers and subsequently increased student learning.

\section{Conclusion}

This qualitative study explored the long-term impact of a faculty development program. The study focused on participants from the University of Missouri's New Faculty Teaching Scholars program five years after the program. Kirkpatrick's (1998) four-level evaluation model was used to gather data for the study. Although the Kirkpatrick model was created to evaluate training programs in business, this research reinforced previous studies that found the model to be an effective tool in evaluating educational programs as well. Through document review, interviews, and observations, the study results provided evidence that the NFTS faculty development program continued to have positive impacts on the participants over time.

\section{REFERENCES}

Amundsen, C., McAlpine, L., Weston, C., Krbavac, M., Mundy, A., \& Wilson, M. (2005). The what and why of faculty development in higher education: An in-depth review of the literature. Retrieved from http://www.sfu.ca /rethinkingteaching/publications/CAmundsen.etal.pdf

Boyle, M. A., \& Crosby, R. (1997). Academic program evaluation: Lessons from business and industry. Journal of Industrial Teacher Education, 34(3), 81-85.

Camblin Jr., L. D., \& Steger, J. A. (2000). Rethinking faculty development [Electronic version]. Higher Education, 39, 1-18.

Davidson-Shivers, G. V., Salazar, J., \& Hamilton, K. H. (2005). Design of faculty development workshops: Attempting to practice what we preach [Electronic version]. College Student Joumal, 39(3), 528-539. 
Guest, G., Bunce, A., \& Johnson, L. (2006). How many interviews are enough? An experiment with data saturation and variability. Field Methods, 18(1), 59-82.

Kirkpatrick, D. L. (1975). Evaluating training programs: $A$ collection of articles from the Journal of the American Society for Training and Development Alexandria, VA: American Society for Training and Development.

Kirkpatrick, D. L. (1998). Evaluating training programs: The four levels (2nd ed.). San Francisco, CA: Berrett-Koehler.

Knight, A. M., Carrese, J. A., \& Wright, S. M. (2007). Qualitative assessment of the long-term impact of a faculty development programme in teaching skills [Electronic version]. Medical Education, 41, 592-600.

Morzinski, J. A., \& Fisher, J. C. (2002). A nationwide study of the influence of faculty development programs on colleague relationships [Electronic version]. Academic Medicine, 77(5), 402-406.

Morzinski, J. A., \& Simpson, D. E. (2003). Outcomes of a comprehensive faculty development program for local, full-time faculty [Electronic version]. Family Medicine, 35(6), 434-439.

Naugle, K. A., Naugle, L. B., \& Naugle, R. J. (2000, Fall). Kirkpatrick's evaluation model as a means of evaluating teacher performance [Electronic version]. Education, 121(1), 135-144.

New Faculty Teaching Scholars. (2004). General goals. University of Missouri: Academic Affairs. Retrieved from http:/www.umsystem.edw/ums/departments /aa/nfts/goals.shtml

Pacheco, M. T. (2001). Design for the future: Creating the learner-centered research University-University of Missouri System Strategic Plan. University of Missouri System: Office of the President.

Patton, M. Q. (1990). Qualitative evaluation and research methods (2nd ed.). Newbury Park, CA: Sage.

Pittas, P. A. (2000). A model program from the perspective of faculty development [Electronic version]. Innovative Higher Education, 25(2), 97-110.

Steinert, Y. (2000). Faculty development in the new millennium: Key challenges and future directions [Electronic version]. Medical Teacher, 22(1), 44-50.

Thomas, R. M. (2003). Blending qualitative and quantitative research methods in theses and dissertations. Thousand Oaks, CA: Corwin Press.

University of Missouri System. (2001-2008). New faculty teaching scholars annual evaluation reports. Unpublished reports. Academic Affairs.

Worthen, R. D., Sanders, J. R., Fitzpatrick, J. L. (1997). Program evaluation: Alternative approaches and practical guidelines (2nd ed.). White Plains, NY: Longman. 